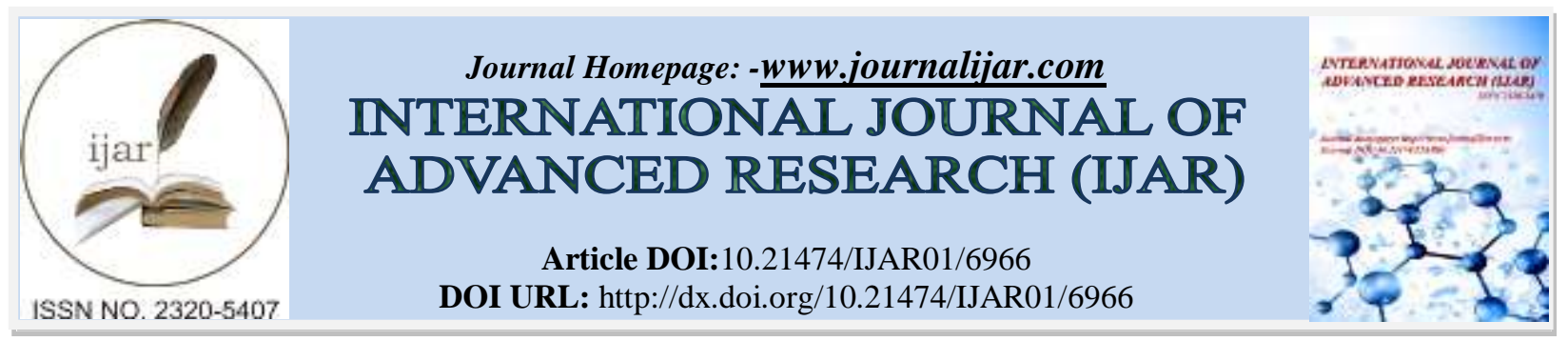

RESEARCH ARTICLE

\title{
PROSPECTIVE RANDOMISED COMPARITIVE STUDY ON EFFECTS OF ADDING DEXMEDETOMIDINE AND FENTANYL TO EPIDURAL BUPIVACAINE ON POST OPERATIVE ANALGESIA IN PATIENTS UNDERGOING LOBECTOMY.
}

1. MD., Professor of Anaesthesiology, Madras Medical College.

2. MD., DA, Professor of Anaesthesiology, Madras Medical College.

\section{Manuscript Info}

Manuscript History

Received: 16 February 2018

Final Accepted: 18 March 2018

Published: April 2018

\section{Abstract}

Aim: To compare the duration and quality of postoperative analgesia between fentanyl and dexmedetomidine when added epidurally to bupivacaine in patient undergoing lobectomy.

Materials and methods: After obtaining institutional ethical approval adult patients aged 18 to 60 years undergoing elective lobectomy with ASA PS II \&III who have given valid informed consent patients were divided into two groups. Group D and group F. Group allocation was done by closed envelope method.

Group D - received $8 \mathrm{ml}$ of $0.125 \%$ bupivacaine and $1 \mathrm{mic} / \mathrm{kg}$ of dexmedetomidine diluted to $2 \mathrm{ml}$ via the epidural catheter.

Group F-received $8 \mathrm{ml}$ of bupivacaine and $1 \mathrm{mic} / \mathrm{kg}$ of fentanyl diluted to $2 \mathrm{ml}$ via epidural catheter.

Result: This double-blinded prospective randomised controlled study was done to evaluate the duration of analgesia as well as sedation and adverse effects of dexmedetomidine $(1 \mathrm{mic} / \mathrm{kg})$ and fentanyl $(1 \mathrm{mic} / \mathrm{kg})$ given via epidural route with $0.125 \%$ bupivacaine in patients postoperatively who underwent lobectomy surgeries under general anaesthesia. This study analysed the duration of postoperative analgesia, quality of analgesia, the side effects and the hemodynamic changes which showed that dexmedetomidine was better than fentanyl in many aspects.

Conclusion: In conclusion dexmedetomidine appears to have a beneficial effect as an adjuvant to local anaesthetic by improving the quality and increasing the duration of analgesia without the side effects associated with opioids like respiratory depression. It has minimal side effects and good hemodynamic stability, however its routine use in epidural route requires caution with regard to its effects due to central sympatholytic property and further studies are necessary to quantify the required dosage.

Copy Right, IJAR, 2018,. All rights reserved.

\section{Introduction:-}

Thoracotomy is one of the most painful surgeries. Post lobectomy pain leads to delayed recovery and contributes to post-operative morbidity in a highly significant manner. Acute pain on the site of incision alters chest wall 
mechanics and impedes effective expansion of the chest, cough and ability to clear secretions predisposing the patients to delayed recovery, respiratory infection, ventilation perfusion mismatch and hypoxemia due to atelectasis. Optimum pain relief after lobectomy is necessary in order to reduce the incidence of atelectasis and post-operative pneumonia. Epidural analgesia has emerged to be the gold standard analgesic technique for the management of postoperative pain after thoracotomy.

This study was undertaken to compare the effects of adding dexmedetomidine and fentanyl to epidural bupivacaine on post thoracotomy pain characteristics.

Thoracotomy requires a very painful muscle splitting incision that is subject to continuous motion as the patient breaths. The reason for severe pain after thoracotomy is due to retraction, resection or fracture of rib, splitting of muscles like latissimus dorsi, serratus anterior, pectoralis major and the intercostal muscles, dislocation of costovertebral joint, irritation of pleura by intercostal drainage tubes and injury to intercostal nerves.

Suboptimal management of pain after thoracotomy has major respiratory consequences. Inspiration is limited by pain, leading to decreased functional residual capacity, atelectasis shunting and hypoxemia. Moreover deep breathing requires stretching of the incision sight, patients without adequate pain relief try to prevent stretching of the incision by contracting there expiratory muscles i.e. splinting, so as to limit the stretch occurring at the incision during inspiration. This failure to take a deep breath before exhaling forcefully result in ineffective cough leading to retention of secretions, atelectasis and pneumonia leading to respiratory failure. Analgesic options available include central neuraxial or regional anaesthesia (epidural, intrathecal and paravertebral)

\section{Aim and Objective:-}

To compare the duration and quality of postoperative analgesia between fentanyl and dexmedetomidine when added epidurally to bupivacaine in patient undergoing lobectomy.

To evaluate hemodynamic changes between the two groups, sedation and adverse effects, if any.

\section{Materials and Methods:-}

Exclusion Criteria:-

1. Patients younger than 18 years or older than 60 years

2. Known allergy to bupivacaine or anaesthetic drugs

3. Patients with deformities of vertebral column

4. Patients with hepatic or renal insufficiency

5. Patients with neurological or psychiatric illness

6. Patients with coagulation disorders

7. Patients with BMI more than $35 \mathrm{~kg} / \mathrm{m}^{2}$

8. Patients with pre-existing motor or sensory deficits

9. Patients with predicted postoperative $\mathrm{FEV}_{1}<40 \%$

50 adult patients undergoing elective lobectomy and planned for epidural analgesia postoperatively were included in this randomised double-blinded study. They were randomly allocated into two groups Group D and group F.Group allocation was done by closed envelope method.

Group D- received $8 \mathrm{ml}$ of $0.125 \%$ bupivacaine and $1 \mathrm{mic} / \mathrm{kg}$ of dexmedetomidine diluted to $2 \mathrm{ml}$ via the epidural catheter.

Group F-received $8 \mathrm{ml}$ of bupivacaine and $1 \mathrm{mic} / \mathrm{kg}$ of fentanyl diluted to $2 \mathrm{ml}$ via epidural catheter.

\section{Discussion:-}

On the day of surgery basic monitors like ECG pulse oximeter and NIBP were connected. Patience were premedicated and they were put in the right lateral position, $16 \mathrm{G}$ Tuohy's needle was introduced into the T6-7 or T7-8 interspinous space. In all patients the midline approach was used to enter into the interspinous space. $3 \mathrm{ml}$ of lignocaine $1.5 \%$ test dose within 1 in 2,00,000 adrenaline was given. Procedure was considered as a failure if, there was unsatisfactory post-operative analgesia with a VAS greater than 4 at the first assessment at 15 mins after giving the block or after extubation. 
Post operatively, patient's vital parameters were assessed every 15 mins in the $1^{\text {st }}$ hour, every half an hour in the second hour and then hourly for 6 hrs.

Table 1:-Comparison of mean duration analgesia

\begin{tabular}{|l|l|l|l|}
\hline Group & $\begin{array}{l}\text { Mean duration of } \\
\text { analgesia }\end{array}$ & Standard deviation & P value \\
\hline D & 329.92 & 23.058 & $<0.001$ \\
\hline F & 110 & 9.345 & \\
\hline
\end{tabular}

The mean duration of analgesia was significantly longer in dexmedetomidine group than fentanyl group which was statistically significant.

Table 2:-Comparison of postoperative visual analogue scores

\begin{tabular}{|l|l|l|l|}
\hline TIME(in mins) & GROUP D & GROUP F & P VALUE \\
\hline 30 & 0.00 & 1.12 & $<0.001$ \\
\hline 45 & 0.04 & 1.20 & $<0.001$ \\
\hline 60 & 0.32 & 2.00 & $<0.001$ \\
\hline 90 & 0.60 & 2.40 & $<0.001$ \\
\hline 120 & 1.24 & 3.76 & $<0.001$ \\
\hline 180 & 1.76 & - & - \\
\hline 240 & 2.32 & - & - \\
\hline 300 & 3.16 & - & - \\
\hline 360 & 3.92 & - & - \\
\hline
\end{tabular}

VAS in dexmedetomidine group were 0 in $1^{\text {st }}$ one and half hrs and were between $1-2$ in next 2 hrs and $3 \mathrm{hrs}$ thereafter. The differences in VAS between the 2 groups at all the time intervals were statistically highly significant.

Table 3:-Comparison of sedation scores

\begin{tabular}{|l|l|l|l|}
\hline Time & Group d & Group f & P value \\
\hline 15 & 3 & 2 & $<0.001$ \\
\hline 30 & 3 & 1 & $<0.001$ \\
\hline 45 & 3 & 1 & $<0.001$ \\
\hline 60 & 3 & 1 & $<0.001$ \\
\hline 90 & 2 & 1 & $<0.001$ \\
\hline 120 & 2 & 1 & $<0.001$ \\
\hline 180 & 2 & - & - \\
\hline 240 & 1 & - & - \\
\hline 300 & 1 & - & - \\
\hline 360 & 1 & - & - \\
\hline
\end{tabular}

Median sedation scores were significantly higher in dexmedetomidine group compared with fentanyl group. No patients in either of the groups has sedation score of 4 or 5 at any point of time.

\section{Summary:-}

This double blinded prospective randomized study was done to evaluate the duration of analgesia as well as sedation and adverse effects of dexmedetomidine and fentanyl given via epidural route with $0.125 \%$ bupivacaine in patients post operatively who underwent lobectomy under general anaesthesia.

The following observations were made:

1. The addition of dexmedetomidine to $0.125 \%$ bupivacaine significantly prolonged the duration of postoperative analgesia compared to fentanyl.

2. The quality of analgesia was significantly better when dexmedetomidine was added to bupivacaine rather than fentanyl.

3. The addition of dexmedetomidine epidurally produced sedation that was arousable for many hours.

4. The incidence of side effects such as hypotension, bradycardia, nausea, vomiting and pruritis was not significant between two groups.

5. No episode of respiratory depression was note in both the study groups which are more common with opioids.

6. Hemodynamic stability was well maintained in patients belonging to both the groups. 


\section{Conclusion:-}

In conclusion dexmedetomidine appears to have a beneficial effect as an adjuvant to local anaesthetic by improving the quality and increasing the duration of analgesia without the side effects associated with opioids like respiratory depression. It has minimal side effects and good hemodynamic stability, however its routine use in epidural route requires caution with regard to its effects due to central sympatholytic property and further studies are necessary to quantify the required dosage.

\section{Bibliography:-}

1. S,AroraV,kaur J, Singh A, parmarSS.comparitive evaluation of dexmedetomidine and fentanyl for epidural analgesia in lower limb orthopaedic surgeries-Saudi J Anaesth.

2. Lee's synopsis of anaesthesia.13 ${ }^{\text {th }}$ edition.2006.section 4.1.local anaesthetics.

3. Sabanathan S, Eng J, MearmsAJ,.Alteration in respiratory mechanics following thoracotomy. JRcollsurgedinb 1990;

4. OchrochEA,Gottschalk A. impact of acute pain and its management for thoracic surgical patients. thoracsurgclin 2005.

5. Morgan GE Jr,MikhailMSM,MurrayMJM:clinical anaesthesiology $3^{\text {rd }}$ ed.mc graw-hill,2002.

6. CousinsM,Veering B:epidural neural blockade. In cousins M,Bridenbaugh P:neiral blockade, $3^{\text {rd }}$ ed.lippincottraven,1998,PP 243-320.

7. JinD,KahnRM,kumarD. Perioperative effect of epidural dexmedetomidine with intrathecal bupivacaine on hemodynamic parameters and quality of analgesia.southafr J anaesthanalg 2012;

8. Saadawy I, bokerA,ElshahawyMA,AlmazrooaA.effects of dexmedetomidine on the characteristics of bupivacaine in a caudal block in paediatrics.acta anaesthesiology scandinavica 2009;

9. Licker M, SpiliopoulosA,tschoppJM.Influenze of thoracic epidural analgesia on CVS autonomic control after thoracic surgery.Br J anaesth 2003.

10. Sudeeshk,Hasoor SS. Dexmedetomidine in anaesthetic practice:Indian journal of anaesthesia.2011;55(4):323324. 